\title{
Capsule Commentary on Velez et al., "It's Been an Experience, a Life Learning Experience": A Qualitative Study of Hospitalized Patients with Substance Use Disorders
}

\author{
Maria G. Frank, MD FACP FHM \\ Denver Health Hospital Authority, Denver, CO, USA. \\ J Gen Intern Med 32(3):314 \\ DOI: $10.1007 / \mathrm{s} 11606-016-3954-1$ \\ (c) Society of General Internal Medicine 2016
}

$\mathrm{V}$ elez and colleagues ${ }^{1}$ describe a qualitative analysis of hospitalized adults with substance use disorder (SUD) and their perception of factors affecting their motivation to start medication-assisted treatment (MAT). Based on interviews of 32 patients, the authors conclude that the main barriers to initiating MAT during hospitalization were care coordination, patients' feeling of having a "choice" in whether to start treatment, and non-judgmental staff members. The authors stress that hospitalization is an opportunity to initiate treatment for patients with SUD. Although the study's objective was to "explore the experiences of hospitalized adults with SUD and to better understand patient and system factors impacting readiness to change," only the factors as perceived by patients were described.

Suzuki et al. ${ }^{2}$ found that almost half of patients who were started on buprenorphine while hospitalized continued outpatient treatment. Pollini and colleagues ${ }^{3}$ described SUD patients' motivation for substance abuse treatment, readiness to change, and pain and withdrawal symptoms. The authors enrolled 353 patients hospitalized for an acute illness, and identified female gender, being on probation or parole, bipolar disorder, being "tired of using," and family concerns as independent factors associated with a "higher stage of change." O'Toole et al. ${ }^{4}$ found that patients who participated in a day hospital program had increased ambulatory care and decreased emergency visits after discharge, with no effect on re-hospitalization. Pecorano and colleagues ${ }^{5}$ demonstrated that the transition to outpatient MAT during a hospitalization resulted in fewer inpatient medical and behavioral health/substance abuse admissions and emergency department visits, as well as we 11 as a higher number of outpatient behavioral health/ substance abuse admissions, for a small cohort of patients.
Velez and colleagues' interesting and timely article highlights both intrinsic and extrinsic factors as perceived by the patients that led to their willingness to undergo MAT. These results are similar to those of previous studies. Most importantly, all the above-mentioned studies emphasize the importance of engaging SUD patients in treatment while hospitalized. It is imperative that studies analyze how extrinsic factors (provider, society, healthcare system, other) affect access to and engagement in treatment among hospitalized SUD patients. A qualitative study involving provider and health system administrators would likely identify different barriers or determinants (positive or negative) that should be taken into account when considering offering MAT to SUD inpatients.

Corresponding Author: Maria G. Frank, MD FACP FHM; Denver Health Hospital Authority, Denver, CO, USA (e-mail: maria. frank@dhha.org).

\section{Compliance with Ethical Standards:}

Conflict of Interest: The author has no conflicts of interest with this article.

\section{REFERENCES}

1. Velez CM, Nicolaidis C, Korthuis PT, Englander $\mathbf{H}$. "It's been an experience, a life learning experience": a qualitative study of hospitalized patients with substance use disorders. J Gen Intern Med. doi:10.1007/ s11606-016-3919-4.

2. Suzuki J, DeVido J, Kalra I, Mittal L, Shah S, Zinser J, Weiss RD. Initiating buprenorphine for hospitalized patients with opioid dependence: a case series. Am J Addict. 2015;24:10-4.

3. Pollini RA, O'Toole TP, Ford D, Bigelow G. Does this patient really want treatment? Factors associated with baseline readiness to change among hospitalized substance using adults interested in treatment. Addict Behav. 2006;31:1904-18.

4. O'Toole TP, Pollini RA, Ford DE, Bigelow G. The effect of integrated medical-substance abuse treatment during an acute illness on subsequent health services utilization. Med Care. 2007;45(11):1110-5.

5. Pecorano A, Horton T, Ewen E, et al. Early data from project engage: a program to identify and transition medically hospitalized patients into addictions treatment. Addict Sci Clin Pract. 2012;7(20):1-7. 